\title{
ESTADÍSTICA BÁSICA PARA GRUPOS MASIVOS DE ESTUDIANTES: DEMOCRATIZAR LA CALIDAD DE LOS APRENDIZAJES
}

\author{
Eduardo León Bologna \\ Universidad Nacional de Córdoba, Argentina \\ ebologna@gmail.com
}

\begin{abstract}
Se describen estrategias para un curso instrumental de estadística básica en primer año de Psicología. Con un enfoque constructivista, los alumnos se involucran en el proceso de producción y análisis de datos, comparten y comparan sus resultados con los de sus compañeros, y discuten entre pares los procedimientos aplicados y los resultados alcanzados. El desafio es hacerlo con grupos grandes y diversos de estudiantes, y lograr que no solo quienes provienen de las mejores escuelas o de los sectores sociales más favorecidos se beneficien de este enfoque. Para lograrlo, todos los alumnos aplican el mismo instrumento y analizan los datos que producen, los comparan con los de sus compañeros y con resultados internacionales. Los errores que aparecen en estas actividades son retroalimentación para mejorar las actividades prácticas propuestas. Se espera que además de aprender conceptos y procedimientos de análisis, desarrollen actitudes positivas hacia la estadística.
\end{abstract}

\section{INTRODUCCIÓN}

Enseñar estadística es una fuente inagotable de desafíos y lo es más cuando se trata de cursos obligatorios "de servicio" en carreras no técnicas como las de ciencias sociales, humanas o de la salud, cuando los alumnos serán usuarios ocasionales de la estadística (Nicholls, 2001). Hay consenso en que en estos casos la enseñanza de la estadística debe centrarse en datos reales y en el razonamiento estadístico y no en la presentación de una gran cantidad de métodos ni basado en la teoría (Gal \& Ginsburg 1994). El reporte Gaise (citado por Lee y Famoye, 2006) subraya la importancia del uso de datos reales, de la comprensión conceptual en lugar del mero conocimiento de procedimientos, de la actividad que los alumnos realizan durante las clases, del uso de la tecnología para el análisis de los datos y para apropiarse de las técnicas, y del uso de la evaluación para apoyar los procesos de enseñanza aprendizaje. Se enfatiza la necesidad de aprendizajes activos, estrategias que estimulen la participación de los alumnos, como proyectos, discusiones grupales, análisis hechos por ellos mismos sobre datos reales que hayan recolectado.

La implementación de estas recomendaciones encuentra limitaciones de diferente orden dependiendo del contexto en que se pretenden aplicar; en el caso que aquí se presenta, los problemas son: gran tamaño de los grupos de alumnos, disparidad de nivel de formación previa, escaso equipamiento tecnológico disponible.

\section{PSICOESTADÍSTICA}

En el primer año de la carrera de Psicología de la Universidad Nacional de Córdoba se dicta la materia Psicoestadística Descriptiva e Inferencial, con los inconvenientes propios de todos los cursos de servicio de estadística más algunas particularidades:

- Aunque actualmente la facultad hace esfuerzos e inversiones en fortalecer la investigación básica y aplicada, hay una orientación históricamente clínica de la carrera. Como consecuencia, los alumnos que llegan a estudiar Psicología tienen en mente la imagen del profesional en su consultorio y en muy alta proporción se inclinan hacia la psicología clínica, en sus múltiples variantes.

- La materia Psicoestadística se ubica en el primer año de la carrera. Esto es así porque en el plan de estudios, en segundo año cursan Técnicas Psicométricas, para las que estadística es un insumo y además Psicología experimental y neuropsicología que exigen interpretar resultados de experimentos que se leen en la bibliografía, la mayoría de los cuales están expresados en lenguaje estadístico.

- Gran diferencia con la manera de estudiar las otras materias de primer año, que requieren lectura, memorización y un pensamiento holístico. Por el contrario, estadística se aprende a través de aplicaciones que los mismos alumnos deben intentar, equivocarse, consultar hasta 
apropiarse de los procedimientos. Decidir qué análisis corresponde según el problema y los tipos de variables, cómo solicitar ese análisis a un software y como leer los resultados.

- Los grupos de alumnos que ingresan cada año a la carrera son numerosos, entre 1500 y 2000. Porque no hay restricciones para el ingreso, salvo la de haber concluido la escuela media.

- Por características del sistema educativo en Argentina, este conjunto de estudiantes es heterogéneo en su formación previa, que va desde ninguna exposición a contenidos estadísticos a lo largo de la escuela media, hasta algunos que cuentan con conocimientos básicos de probabilidad y de medidas descriptivas.

Aun con estos condicionantes, esperamos aproximarnos a que nuestro curso genere aprendizajes significativos y duraderos y que dé lugar a una actitud positiva hacia la estadística que posibilite profundizar más tarde (Garfield et al., 2002), porque hay una porción creciente de estudiantes que más tarde eligen la investigación, para la que necesitarán al menos rudimentos de pensamiento estadístico (Chance, 2002, Wild \& Pfannkuch, 1998). Para lograr esto, la decisión de introducir cambios tuvo como referencia a la teoría constructivista (Vygotsky, 1978), dado que hay consenso (Kim, 2005; Hassad, 2007; Hassad, 2011; Senoc, 2014) en que los enfoques basados en ella llevan a los estudiantes a involucrarse en el proceso de enseñanza-aprendizaje e impactan positivamente en el modo en que se relacionan con los contenidos.

El punto de partida para la implementación de cambios es la estrategia didáctica tradicional en la materia, que consistió en clases teóricas expositivas y clases prácticas dedicadas a la resolución de ejercicios de aplicación. Dado que no se contaba con equipamiento informático y no podía asegurarse que todos los alumnos tuvieran acceso a él, la atención estaba puesta en la elección de las técnicas adecuadas a determinado objetivo de análisis y en la interpretación de resultados, sin aplicación de los procedimientos. Por la imposibilidad de generar datos propios, se usaban los provenientes de investigaciones publicadas, o bien ficticios presentados en situaciones artificiales. Esto se decidió porque los alumnos de primer año no cuentan aún con conceptos psicológicos a los que puedan aplicarse los análisis.

Antes de implementar reformas es necesario conocer las dificultades que encuentran los alumnos (Garfield y Ahlgren, 1988; Ghinis et al., 2009). La observación de los procesos de aprendizaje a lo largo de los últimos diez años mostró que algunas dificultades eran semejantes a las que se encuentran cada vez que se dictan cursos de servicio de Estadística, pero que hay otras que se derivan de los condicionantes señalados y son específicas de nuestros alumnos:

- Porque la materia está en primer año de la carrera, una dificultad es la perspectiva excesivamente abstracta que los estudiantes hallan en los contenidos, ya que las aplicaciones que se ofrecen no se anclan en conceptos empíricos que les resulten familiares. Cuando se ofrecen ejemplos de investigaciones reales, los alumnos fallan al interpretar los conceptos en juego, y a esta dificultad debe agregarse el aprendizaje de procedimientos estadísticos.

- Porque la educación media en Argentina tiene una oferta de calidad variada, algunos alumnos han incluido en sus aprendizajes preuniversitarios muchos de los conceptos con que se inicia la materia, mientras que para otros son completamente novedosos.

- Por la masividad del alumnado y la carencia de salas de informática suficientes, no se pudieron reproducir los análisis en manos de los alumnos ni usar datos por ellos producidos. Los alumnos sienten una especie de "caja negra" entre la elección del procedimiento y el resultado que deben interpretar.

- Porque una proporción alta de los alumnos tiene en mente la Psicología clínica como horizonte laboral (aunque luego cambie), la idea de trabajar con agregados le resulta ajena. Aun las explicaciones sobre el uso de estadística en las pruebas psicométricas y en investigación, son difíciles de aceptar. Psicología aparece como ciencia de los individual, de los subjetivo y resulta difícil comprender la necesidad de obtener generalidad en los resultados de investigación. 
El desafío era entonces el de disponer de mecanismos de enseñanza y de evaluación con los que se logre una apropiación de los contenidos suficiente para los usos posteriores y en la medida de lo posible para todos los alumnos. Esto último es importante, porque por la disparidad de la formación previa, todos los años hay grupos de alumnos que superan la materia exitosamente y sin dificultades, cuando otra proporción importante fracasa. Además, la Universidad Nacional de Córdoba se plantea entre sus objetivos el de la inclusión de alumnos provenientes de diferentes sectores sociales; así, cualquier solución que se proponga debe ser accesible masivamente.

\section{PRIMERA ETAPA DE CAMBIOS}

En una primera etapa de reforma en el dictado, se avanzó en la resolución de algunas de las dificultades por medio de la elaboración de un libro exclusivo para estos estudiantes, con formato de manual. En el libro participan integrantes de la cátedra, de modo que el estilo de la bibliografía es cercano a lo que los alumnos hallan en las clases, como lo recomiendan Peiris y Beh (2006). Además, se graduó la dificultad de los exámenes parciales, de los que el primero es muy elemental, para que los estudiantes ganen en confianza sobre sus propias habilidades. Se han elaborado vídeos tutoriales para el uso del paquete estadístico Infostat (Di Rienzo, 2012), y se ha promovido su uso, pero sin establecerlo como condición para aprobar la materia, dado que no todos los alumnos pueden acceder a una computadora. Estas reformas han permitido que la materia se vuelva más amigable y no corra el riesgo de ser llamada "sadística" (Rosenthal, 1992; Forte, 1995), lo que debe considerarse de importancia, ya que reducir la ansiedad frente a la materia contribuye a facilitar su aprendizaje (Chiesi y Primi, 2010), en especial en alumnos que tienen trayectorias de frustración en materias afines.

Aun con estos cambios, los resultados que se obtienen no alcanzan a ser satisfactorios: una alta proporción de alumnos que inician el cursado, abandona antes del primer parcial y, aquellos que aprueban la materia hallan grandes dificultades para transferir los contenidos al uso práctico, cuando deben usarlos en la puesta a punto de pruebas psicométricas, o para interpretar resultados de investigaciones expresados en lenguaje estadístico, un año más tarde.

\section{SEGUNDA ETAPA: LA PROPUESTA}

En esta nueva etapa se busca que los estudiantes:

- Se comprometan en las actividades, por medio de la realización de análisis reales con datos que les sean familiares. El intento por aprender técnicas de análisis aplicándolas a conceptos desconocidos duplica el nivel de dificultad.

- Compartan y comparen los resultados con sus compañeros y con otras fuentes, de modo que vayan construyendo el sentido sustantivo de los resultados estadísticos que obtienen de los análisis.

- Ganen confianza en sus capacidades de manera gradual, para superar los prejuicios sobre la dificultad de la materia y sus dificultades personales frente a contenidos altamente formalizados, cercanos a la matemática.

- Puedan estudiar de manera autónoma, de modo que regulen los tiempos y los adapten a los diferentes ritmos de aprendizaje, atendiendo a la diferente formación previa de que disponen desde la escuela media.

Aunque estos cambios no parecieron viables hasta hace poco tiempo, en la actualidad se cuenta con algunos medios disponibles:

- Programa nacional Conectar Igualdad (http://www.conectarigualdad.gob.ar), que distribuye netbooks entre estudiantes de escuelas públicas de nivel medio de Argentina.

- Software de análisis de datos amigable y gratuito para los alumnos: el paquete Infostat/E.

- Material didáctico elaborado por los docentes: manual de contenidos teóricos, con aplicaciones y actividades prácticas, y vídeos tutoriales para ilustrar el uso del software.

Estas facilidades hacen viable que se reproduzcan situaciones reales de análisis de datos, ya que una porción grande de alumnos cuenta con una computadora portátil a la que pueden equipar con el software. Además, los alumnos pueden avanzar a su propio ritmo, usando los materiales didácticos que se ofrecen. 
La propuesta consiste en la conformación de grupos de entre seis y ocho alumnos que trabajarán juntos durante la materia. Cada alumno aplica el mismo cuestionario a 10 casos y genera sus datos, a los que carga en una base propia del grupo. Cada grupo tiene su base y puede comparar los resultados de sus análisis con los de los otros grupos. Estas bases se suben al aula virtual y luego se compilan en una base única de todo el curso, que se utiliza para ejemplos generales.

\section{Datos propios}

La primera dificultad para disponer de datos propios es que no han cursado aun materias sobre metodología de la investigación y no pueden construir un instrumento de calidad, por lo que se decide ofrecer un cuestionario elaborado por los docentes. Éste debió ser de muy sencilla administración, que evitara preguntas sensibles a sesgos de aplicación (por ejemplo, se necesitan encuestadores bien entrenados para indagar sobre hábitos ilegales). Los conceptos que incluyera el cuestionario debían ser fácilmente accesibles desde el sentido común; ser cercanos a la propia experiencia, para que su dificultad no distraiga del foco en los procedimientos estadísticos. Al mismo tiempo debía tener referencias teóricas de interés psicológico. Y en el conjunto de ítems, que requería que hubiese variables de diferente nivel de medición a fin de practicar los distintos procedimientos.

Teniendo estas consideraciones en cuenta, se elaboró un cuestionario de diez ítems que contienen: dos conceptos relevados en la Encuesta Mundial de Valores (WVS, http://www.worldvaluessurvey.org), tres ítems sobre uso del tiempo y datos de filiación. Los dos primeros son: el control que el individuo considera tener sobre su propia vida y su preferencia por valores materialistas o postmaterialistas (Roser, 2014). Las preguntas sobre el uso del tiempo se refieren a la dedicación al estudio, al trabajo y la recreación.

El trabajo comienza con la aplicación del cuestionario a una muestra no probabilística de estudiantes universitarios. Se restringe así la población objetivo a fin de reducir la variabilidad de los resultados y facilitar las comparaciones con muestras pequeñas. Luego cada grupo crea su propia base de datos. En las clases prácticas (dos horas por semana) se dispone de una guía que orienta el análisis de los datos recogidos. La primera etapa es de descripción (tablas, gráficos, medidas resumen) y la segunda inferencial (estimación por intervalos y pruebas de hipótesis). Los resultados que cada grupo obtenga se comparan con los de los demás grupos. Las respuestas obtenidas a las preguntas de la WVS pueden también ser comparadas con las dadas por los diferentes grupos a los que se encuesta internacionalmente, con la salvedad de las condiciones en que fueron tomadas las muestras propias y la población a la que se dirigieron.

\section{Graduación de los aprendizajes}

Los contenidos son expuestos por primera vez a los estudiantes en las clases teóricas, y disponen del manual que usa el mismo vocabulario y forma de presentación de los temas. Paralelamente, han aplicado el cuestionario y deben subir la base al aula virtual para que sea controlada en su calidad y para contar con resguardo de los datos. En las clases prácticas, se ilustran los procedimientos usando Infostat/E. Luego, los alumnos reproducen los análisis sobre sus propios datos, para lo que deben contar con al menos una computadora por grupo. Los docentes y el personal de apoyo recorren los grupos monitoreando los avances y las dificultades y ayudando a resolverlas. Al final de la clase se exponen y comparan los resultados y se explican para todos las principales dificultades halladas. Esto puede no ser suficiente para todos los alumnos, por su diferente formación previa y porque los grupos son numerosos. Para reforzar estas prácticas, los alumnos disponen en el aula virtual con un conjunto de vídeos tutoriales que muestran cómo se realiza cada análisis con el paquete elegido.

Al finalizar la parte descriptiva deben subir un trabajo de síntesis y nuevamente al cabo de la parte inferencial. Estos trabajos son evaluados y, junto con los exámenes parciales, hacen a la condición de regular.

Los errores que se cometen en las actividades prácticas y los trabajos de síntesis son registrados por los docentes a fin de entender los modos de razonamiento, usualmente de sentido común, que conducen a ellos. Estos registros son insumo valioso para ajustar los materiales didácticos y las clases: modificar el manual, dedicar más tiempo a la explicación de algunos temas, reforzar los recursos audiovisuales. 
Se mantiene la graduación en la dificultad de los parciales, como forma de fomentar la confianza en las propias capacidades, alcanzando pequeños logros. Razones de programación impiden que se aumente el número de exámenes parciales de tres a cinco, pero en las actividades prácticas semanales se realizan aplicaciones de dificultad creciente. Como lo sugiere Marson (2007) se intenta que los alumnos obtengan una retroalimentación inmediata de sus intentos, y esto sucede durante los prácticos.

\section{Evaluación parcial}

Los resultados de la evaluación de esta reforma estarán disponibles al final de 2015 cuando pueda compararse el nivel de logro de los alumnos con los valores medios históricos de la materia. La comparación se hará de manera cuantitativa sobre la proporción de aprobados, de promocionados y de quienes abandonan el cursado. Nuestro equipo aplicó en los años 2011 y 2012 una escala sobre actitudes hacia la estadística (Bologna \& Vaiman, 2013), cuyo resultados podrán compararse con los que se alcancen luego de esta reforma. Además, se prevé entrevistar a alumnos que hayan tenido diferente grado de logro, para conocer la experiencia subjetiva con las actividades propuestas.

Al momento actual se ha realizado una exploración del impacto, usando grupos pequeños y comparando las lecturas de resultados que hacen alumnos que trabajaron con la estrategia tradicional y con la nueva. No es posible usar un diseño experimental con grupos al azar, por lo que deberán compararse resultados entre grupos de años diferentes. La comparación se realiza con estudiantes de Psicología también, pero de una universidad privada, las condiciones son diferentes, pero es útil como primera exploración. Durante el cursado de 2013 se solicitaron trabajos prácticos basados en el análisis de datos provenientes de una base pública: el Operativo Nacional de Evaluación Educativa. Se trata de un estudio de cobertura nacional que recoge información sobre aspectos socioeconómicos y educativos de los hogares de alumnos del último curso de escuela media, y los resultados alcanzados en pruebas estandarizadas de matemática y lengua. El año siguiente, los alumnos generaron sus propios datos a través de la aplicación de un cuestionario y basaron en ellos sus análisis.

Para comparar los niveles de logro, se analizó la comprensión de los conceptos de variable, categoría y unidad de análisis. Los alumnos que usaron sus propios datos produjeron informes más completos de los resultados. Se ve mayor proximidad con las variables que se describen y las lecturas no están limitadas a la salida estadística, sino puestas en un contexto que les resulta familiar. En lo inferencial se notan más diferencias, se alcanzó claridad en el concepto de representatividad de la muestra: por haber estado en la producción de los datos, les resultó visible el problema de los sesgos, pudieron redactar mejor los resguardos acerca de los resultados. La diferencia cualitativa entre la población objetivo y la muestra quedó ilustrada al repasar quiénes habían sido los encuestados. El efecto del tamaño de la muestra se puso en evidencia al observar la amplitud de los intervalos de confianza y la falta de significación estadística de las diferencias al comparar grupos, por la baja potencia de las pruebas.

\section{REFERENCIAS}

Bologna, E., \& Vaiman, M. (2013). Actitudes, experiencia previa y nivel de logro en Estadística en la carrera de Psicología. En J. M. Contreras, G. R. Cañadas, M. M. Gea y P. Arteaga (Eds.), Actas de las Jornadas Virtuales en Didáctica de la Estadística, Probabilidad y Combinatoria (pp. 91-103). Granada, Departamento de Didáctica de la Matemática de la Universidad de Granada. http://www.jvdiesproyco.es/documentos/ACTAS/2\%20Comunicacion\%204.pdf accedido el $12 / 3 / 2015$

Chance, B. L. (2002). Components of Statistical Thinking and Implications for Instruction and Assessment. Journal of Statistics Education, 10(3). Disponible en: http://www.amstat.org/publications/jse/v10n3/chance.html accedido el 12/3/2015

Chiesi, F., \& Primi, C. (2010). Cognitive and non-cognitive factors related to students' statistics achievement. Statistics Education Research Journal, 9(1), 6-26. Disponible en: http://iaseweb.org/documents/SERJ/SERJ9\%281\%29 Chiesi Primi.pdf accedido el 12/3/2015 
Di Rienzo J. A., Casanoves F., Balzarini M. G., Gonzalez L., Tablada M., Robledo C. W. InfoStat versión 2012. Grupo InfoStat, FCA, Universidad Nacional de Córdoba, Argentina. URL http://www.infostat.com.ar

Forte, J. A. (1995). Teaching Statistics Without Sadistics. Journal of Social Work Education, 31(2), 204-218.

Gal, I., \& Ginsburg, L. (1994). The Role of Beliefs and Attitudes in Learning Statistics: Towards an Assessment Framework. Journal of Statistics Education, 2(2). Disponible en: http:/www.amstat.org/publications/jse/v2n2/gal.html accedido el 12/3/2015

Garfield, J. \& Ahlgren, A. (1988). Diffculties in Learning Probability and Statistics. Journal for Research in Mathematics Education. Disponible en: http://www.stat.ucla.edu/ Rakhee/attachments/garfieldahlgren.pdf accedido el 12/3/2015

Garfield, J., Hogg, B., Schau, C., \& Whittinghill, D. (2002). First Courses in Statistical Science: The Status of Educational Reform Efforts. Journal of Statistics Education, 10(2). Disponible en: www.amstat.org/publications/jse/v10n2/garfield.html accedido el 12/3/2015

Ghinis, D., Korres, K., \& Bersimis, S. (2009). Difficulties Greek Senior High School Students Identify in Learning and the Teaching of Statistics: The Case of Experimental and Private High Schools. Journal of Statistics Education, 17(3). Disponible en: http://www.amstat.org/publications/jse/v17n3/ghinis.html accedido el 12/3/2015

Hassad, Rossi A. (2011). Constructivist and Behaviorist Approaches: Development and Initial Evaluation of a Teaching Practice Scale for Introductory Statistics at the College Level, Numeracy, 4(2), Artículo $7 . \quad$ DOI: disponible en: http://scholarcommons.usf.edu/numeracy/vol4/iss2/art7 accedido el 12/3/2015

Kim, J. S. (2005). The Effects of a Constructivist Teaching Approach on Student Academic Achievement, Self-Concept, and Learning Strategies. Asia Pacific Education Review, Vol. 6 N. 1 pags. 7-19. Disponible en: http://files.eric.ed.gov/fulltext/EJ728823.pdf accedido el $12 / 3 / 2015$

Lee, C. \& Famoye, F. (2006). Teaching Statistics Using a Real Time Online Database Created by Students. En ICOTS-7, 1-6. Disponible en: http://iaseweb.org/documents/papers/icots7/7A3_LEEF.pdf accedido el 12/3/2015

Marson, S. M. (2007). Three Empirical Strategies for Teaching Statistics. Journal of Teaching in Social Work, 27(3/4). Disponible en: http://libres.uncg.edu/ir/uncp/f/Three\%20Empirical\%20Strategies\%20for\%20Teaching\%20St atistics.pdf accedido el 12/3/2015

Nicholls, D. (2001). Future Directions for the Teaching and Learning of Statistics at the Tertiary Level. International Statistical Review, 69(1):11-15. Disponible en: https://stat.fi/isi99/proceedings/arkisto/varasto/nich0099.pdf accedido el 12/3/2015

Peiris, S. \& Beh, E. J. (2006). Where statistics teaching can go wrong. CAL-laborate International, 15 , 21-23. Disponible

en: http:/openjournals.library.usyd.edu.au/index.php/CAL/article/view/6030/6681 accedido el $12 / 3 / 2015$

Rosenthal, B. (1992). No more sadistics, no more sadists, no more victims. UMAP Journal, 13, 281-290.

Roser, M. (2014). Materialism and Post-Materialism - Our World in Data. Disponible en: http://ourworldindata.org/data/culture-values-and-society/materialism-and-post-materialism/ accedido el 12/3/2015

Senoc, G. A. (2014). The Efficiency of Three Methods of Teaching on Students' Performance in Introductory Statistics. International Journal of Science and Research. Vol 3 N. 1 pgs. 123127. http://www.ijsr.net/archive/v3i1/MDIwMTM3MzA=.pdf accedido el 12/3/2015

Vygotsky, L. S. (1978). Mind in society: The development of higher mental processes. Cambridge, MA: Harvard University Press

Wild, C. \& Pfannkuch, M. (1998). What is Statistical Thinking? en ICOTS-5. Disponible en http://iase-web.org/documents/papers/icots5/Topic3c.pdf accedido el 12/3/2015 